\title{
The Effect of Heat Treatment on the Mechanical Properties of SAE 1035 Steel
}

\author{
Obiukwu Osita $^{1, a^{*}}$, Udeani Henry ${ }^{2, b}$, Ubani Progress ${ }^{3, c}$ \\ ${ }^{1,2,3}$ Department of Mechanical Engineering, \\ School of Engineering and Engineering Technology, \\ Federal University of Technology, Owerri, Imo State, Nigeria. \\ aobiukwu@futo.edu.ng, bobinech@yahoo.com, 'obinech@gmail.com
}

Keywords: Heat treatment, fatigue, Impact, Hardness, Tensile test, SAE 1035 carbon steel.

\begin{abstract}
The effect of various heat treatment operations (annealing, normalizing, tempering) on mechanical properties of $0.35 \%$ carbon steel was investigated. The change in the value of endurance limit of the material as a result of the various heat-treatment operations were studied thoroughly. It was found that the specimens tempered at low temperature $\left(200^{\circ} \mathrm{C}\right)$ exhibited the best fatigue strength. Microscope was used to characterize the structural properties resulting from different heat treatment processes. The results from the tensile tests impact tests and hardness tests showed that the mechanical properties variate at every heat-treatment conditions. The microstructure of differently heat-treated steels was also studied.
\end{abstract}

\section{Introduction}

Engineering components and structures are regularly subjected to cyclic loading and consequently are prone to fatigue damage, which in most cases start at the surface due to localized stress concentrations caused by machining marks, exposed inclusions or even the contrasting movement of dislocations [1]. As technology has been developed, fatigue has become more prevalent in automobiles, aircraft, turbines, etc. subject to repeated loading and vibration. It is estimated that fatigue is responsible for $80 \%$ to $90 \%$ of all engineering failures [2]. According to [3] fatigue failure involves a multi-stage processes that begins with crack initiation, followed by a progressive crack growth across the part with continued cyclic loading, and finally the sudden fracture of the component or specimen. [4]. Fatemi and Yang [2] reported that there are three commonly recognized forms of fatigue namely; high cycle fatigue (HCF), low cycle fatigue (LCF) and thermal mechanical fatigue (TMF). Improvements in fatigue performance in components are derived primarily by decreasing the surface cyclic tensile stress or by increasing the surface yield stress, thereby increasing the resistance to fatigue crack nucleation. To achieve these goals, common surface modification processes, which often simultaneously increase the surface yield stress and introduce a residual compressive stress to decrease the surface cyclic tensile stress, are based on heat treating. Wei et al. [5] studied the fatigue behavior of $1500 \mathrm{MPa}$ bainite/martensite duplex phase high strength steel. It was observed that fatigue strength increases and fatigue crack threshold gives lower crack propagation. The steel produces a better combination of strength, toughness and fatigue properties when tempered at $370^{\circ} \mathrm{C}$ for 2 hours. Mechanical properties are enhanced as the materials gone through the heat treatment processes [6]. In Zahid et al. [6], specimens corresponding to all heat treatment temperatures showed higher hardness as compared to the annealed specimens of the same steel. In general, quenching and tempering results the optimum fatigue properties in heat treated steels although at a hardness level above about Rc 40 bainitic structure produced by austempering results in better fatigue properties than quenched and tempered structure with the same hardness [7]. The poor performance of the quenched and tempered structure indicated by electron micrographs is the result of stress concentration effects of the thin carbide films which are formed during the formation of martensite in tempering and also the fatigue limits increases with decreasing tempering temperature up to a hardness Rc 45 to Rc 55 which is well explained [8]. Fatigue properties at high hardness level are extremely sensitive to the surface 
preparation, residual stresses, and inclusions. Only a small amount of non-martensitic transformation products can cause an appreciable reduction in fatigue limit [9]. The effect of intercritical heat treatment on the mechanical properties of AISI 3115 steel was investigated by Maleque [10]. The experimental results showed that tensile strength increases but impact strength decreases with increasing intercritical temperature, correspondingly with the increase in amount of martensite in the steel. Influence of cold rolling threads before or after heat treatment on high strength SI grade 12.9 bolts for different fatigue preload conditions was carried out by Horn [11]. Hence, steels are normally hardened and tempered to improve their mechanical properties, particularly their strength and wear resistance. In hardening, the steel or its alloy is heated to a temperature high enough to promote the information of austenite, held at that temperature until the desire amount of carbon has been dissolved and then quenched in a particular medium at a suitable rate [12]. Also, in the hardened condition, the steel should have $100 \%$ martensite to attain maximum yield strength, but it is very brittle too and thus quenched steel is used for very few engineering applications. By tempering, the properties of quenched steel could be modified to decrease hardness and increase ductility and impact strength gradually [13]. The resulting microstructures are bainite or carbide precipitate in a matrix of ferrite depending on the tempering temperature [14]. In this study the effects of selected heat treatment techniques on steel mechanical properties, as controlled by processing, is presented.

\section{Experimental Procedures}

Sample Preparation. $0.354 \%$ carbon steel was selected as a material to study the effect of heat treatment on its fatigue strength. Composition of the steel is summarized in Table 1.

Table 1. Chemical composition of as received Medium Carbon Steel

\begin{tabular}{|c|c|c|c|c|c|c|c|c|c|c|c|c|c|}
\hline $\mathbf{C}$ & $\mathbf{S i}$ & $\mathbf{S}$ & $\mathbf{P}$ & $\mathbf{M n}$ & $\mathbf{N i}$ & $\mathbf{C r}$ & $\mathbf{M o}$ & $\mathbf{V}$ & $\mathbf{W}$ & $\mathbf{C u}$ & $\mathbf{A l}$ & $\mathbf{T i}$ & $\mathbf{F e} \%$ \\
\hline 0.354 & 0.236 & 0.061 & 0.052 & 0.730 & 0.098 & 0.158 & $<0.0001$ & $<0.0001$ & $<0.0001$ & 0.292 & 0.021 & 0.008 & 97.99 \\
\hline
\end{tabular}

Source: Spectroanalysis done at Universal Steels Limited, Lagos

The specimen, which was received in ribbed form, was firstly machined to a standard configuration of $10 \mathrm{~mm}$ and $50 \mathrm{~mm}$ gauge diameter and length respectively as shown in Fig. 1 and Fig.2. A set of specimens was prepared for mechanical tests and microstructural analyses. Tensile test and other mechanical specimens were also produced from the as-received medium carbon steel samples of the same composition.

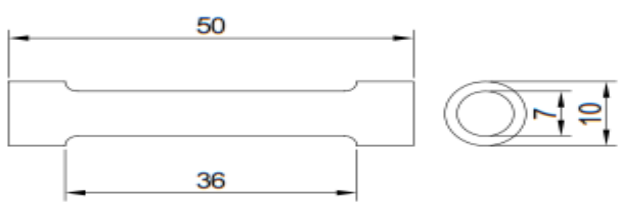

Fig.1. A machined specimen of SAE 1040

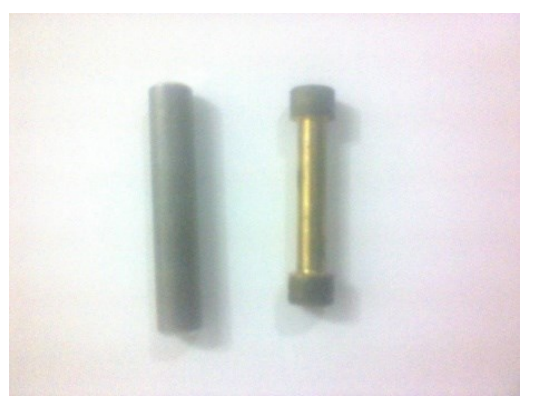

Fig. 2. A Sample of the specimen 
Heat Treatment. Standard heat treatment procedures were adapted to heat treat the carbon steel. Five different samples were prepared for each of the operation. Some of the conventional heat treatments procedure chosen include: annealing, normalizing, tempering and hardening test.

Annealing. The specimen was heated to an annealed temperature of $850^{\circ} \mathrm{C}$. At $850^{\circ} \mathrm{C}$ the samples was held for 1 hour to ensure thorough homogeneity, then the furnace was switched-off so that the furnace and samples temperature gradually decrease to room temperature. The specimen was taken out of the furnace after 48 hours of gradual loss of heat when the furnace temperature would have attain the nominal room temperature.

Normalizing. Each samples of the medium carbon steel to be normalized were placed in the furnace and heated to temperature of $850^{\circ} \mathrm{C}$, was also soaked at that temperature for 1 hour, then allowed to cool in air at a controlled rate- Meanwhile another set of the sample specimens which were not heat treated were taken directly for the tensile test to serve as control samples.

Hardening. The selected samples were heated to $950^{\circ} \mathrm{C}$. At $950^{\circ} \mathrm{C}$ the samples were held for 1 hour to ensure uniform homogeneity. In order to enhance the hardness, the red hot steel is directly and rapidly cooled. Pair of two samples was cooled in automobile engine oil while another pair of two was cooled in water that is at an elevated temperature $40{ }^{0} \mathrm{C}$. This is to avoid quench crack that could ensue if it were cooled at room temperature or below.

Tempering. After quenching the samples from red hot condition to a temperature above room temperature, the samples were subsequently re-heated in the muffle furnace to $200{ }^{\circ} \mathrm{C}$, held for 30 minutes and then air-cooled in order to toughened it and improve on the ductility of the specimens.

Hardness Test. The heat treated specimens were now subjected to hardness test. The hardness of the as received and heat-treated specimens was evaluated using a Brinell hardness (LECO AT700 Micro hardness Tester). Multiple hardness tests were performed on each sample and the average of the best values taken as a measure of the hardness of the specimen.

Tensile Testing. After the successful heat treatment operation, the various heat treated samples were taken for the tensile test. The test was performed on Standard Universal Testing Machine. Tensile tests were conducted at various strain rates of 200, 500, 1000,1500 and $1650 \mathrm{~mm} / \mathrm{min}$ for all the specimens.

Fatigue Test. The fatigue test was done using Avery Denison machine (Avery model 7305). The bending moment was measured. The revolution counter fitted to the motor records the number of stress cycles to failure $(\mathrm{N})$. The calibration curve supplied with the machine was used to show the relationship between dial gauge reading and the imposed torque. The essence of this is to determine the actual load the material in question can withstand before failure in service. The stair case method was used in applying the moment. The applied bending moment was increased by a fixed increment and the next specimen was tested with the new bending moment. The fatigue test of the as received and heat-treated specimens were subjected in to a fatigue testing machine and the specimen were fitted on the two grips tight before the machine start. When the specimen breaks the readings were collected. Fig. 3 shows the Avery Dennison fatigue testing machine. The bending moments imposed were 604, 1208 and $1812.3 \mathrm{MPa}$ for various heat treatments. The bending test was performed at a frequency of $50 \mathrm{~Hz}(1400 \mathrm{rpm})$ for each specimen. It was a complete reversed cycle of stress range $(\mathrm{R})$ and is equal to minimum stress divided by maximum stress which is equal to a negative value $(-1)$ in fatigue tests. 


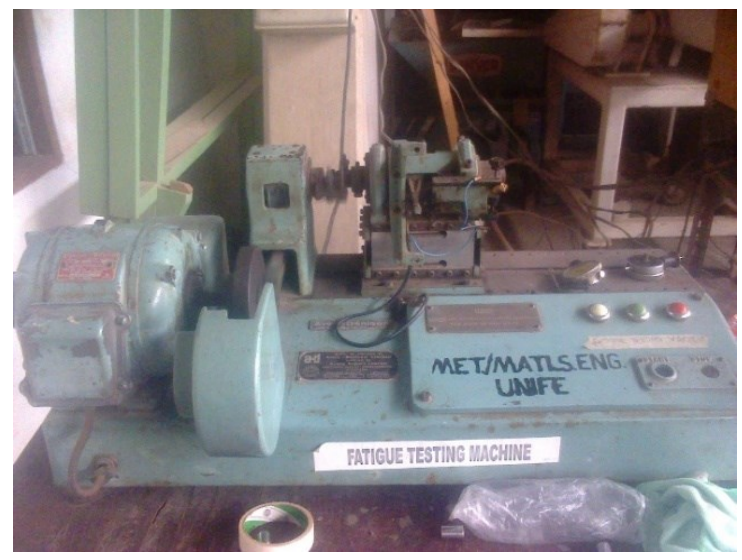

Fig. 3. Avery Denison Fatigue testing machine

Microstructure Examination. Microstructure examination of the as-received and heat-treated specimens was carried out using the Accuscope microscope. Each sample was carefully grounded progressively on emery paper in decreasing coarseness. The grinding surface of the samples were polished using $\mathrm{Al}_{2} \mathrm{O}_{3}$ carried on a micro clothe. The crystalline structure of the specimens were made visible by etching using solution containing $2 \%$ Nitric acids and $98 \%$ methylated spirit on the polished surfaces. Microscopic examination of the etched surface of various specimens was undertaken using a metallurgical microscope with an inbuilt camera through which the resulting microstructure of the samples were all photographically recorded with magnification of 400 .

\section{Results and Discussion}

Microstructural Analysis of the Specimen. Fig. 4 to Fig. 8 show the microstructural view of the specimens. The effects of heat treatment on the microstructures of the samples were studied using the Accuscope microscope. It was observed that the morphologies of the as received samples changed with the heat treatment processes. Some grains are noticed within structures of the samples as the austenitic temperature increased. This significantly alters the orientation of the grains in these samples and it was expected that this change will affect the behavior of these samples when heat treated. From fig. 4, it can be seen that the microstructure comprises of the ferrite (light areas) and pearlite (dark areas) phases of the steel sample. The grain boundaries are even hardly visible due to homogeneity of the constituents in the material.

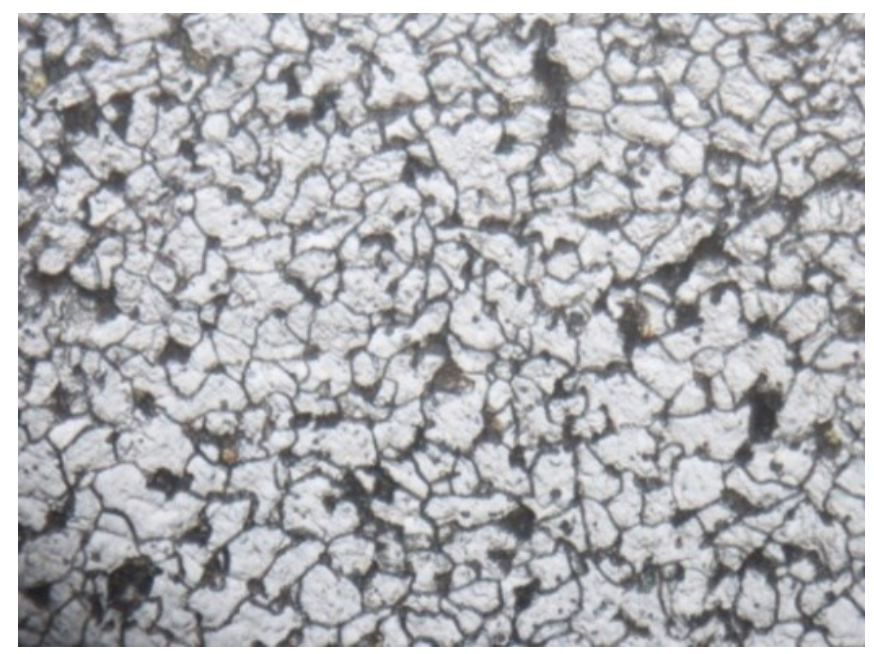

Fig. 4. Microstructural view of the as-received specimen at $360 \mathrm{X}$ 


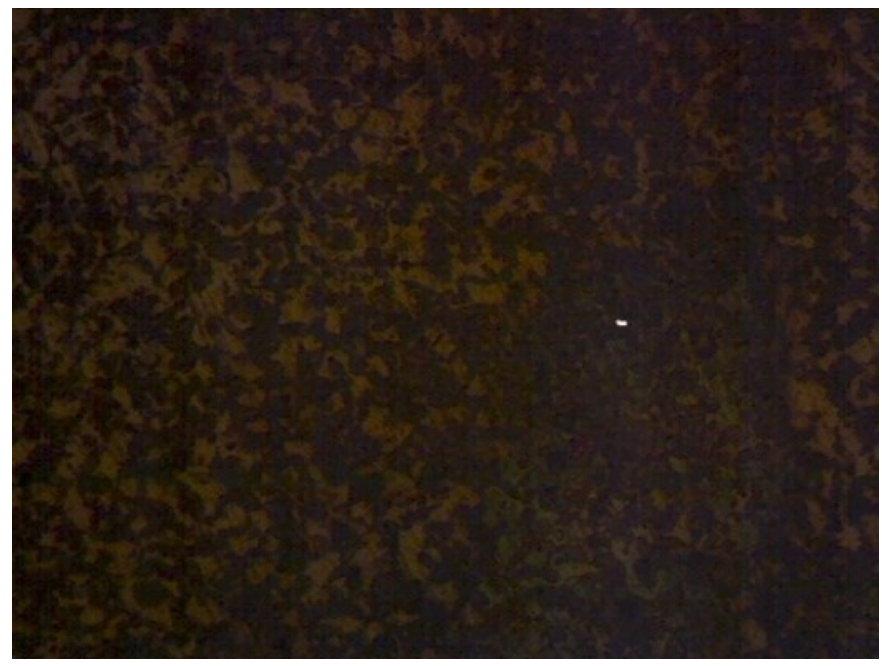

Fig. 5. Microstructural view of the Hardened specimen at 360X

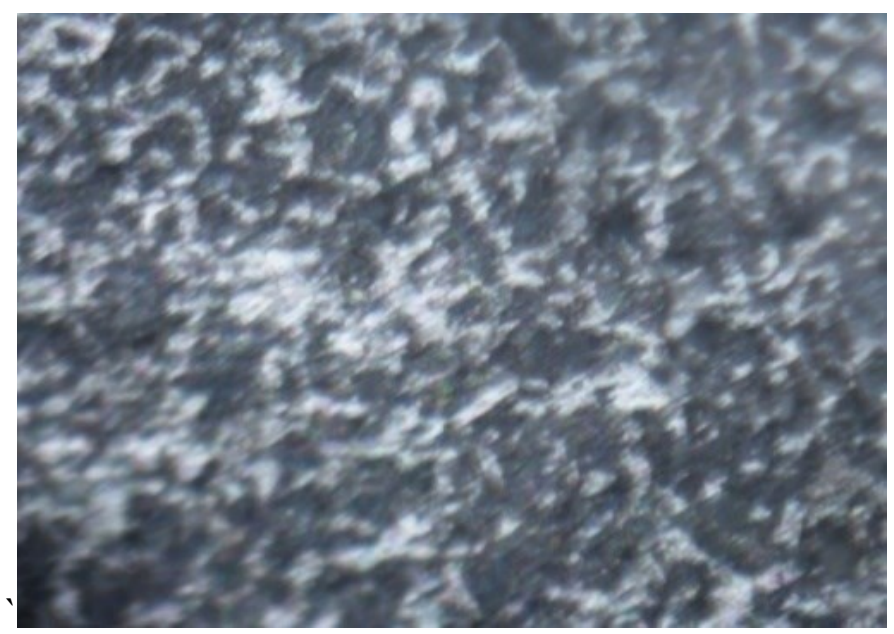

Fig. 6. Microstructural view of the Tempered specimen at 360X

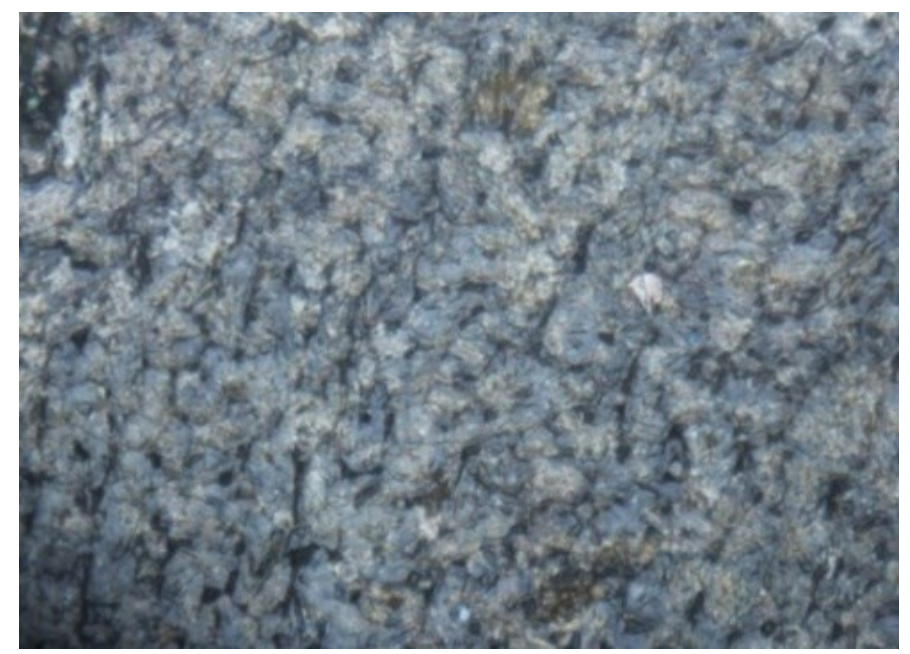

Fig. 7. Microstructural view of the Normalized specimen at 360X 


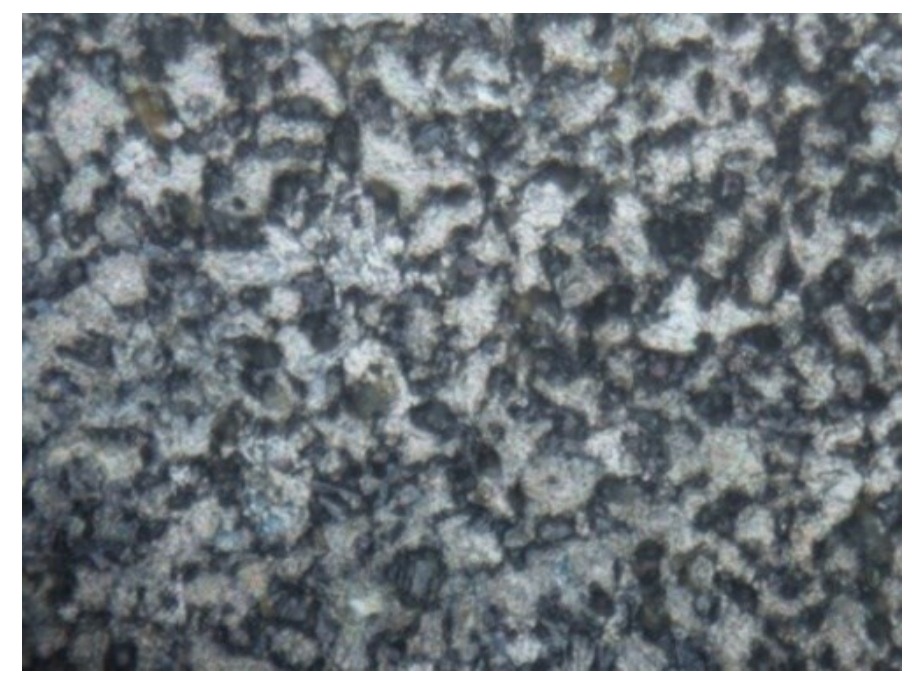

Fig. 8. Microstructural view of the Annealed specimen at $360 \mathrm{X}$

As shown in figures 7 and 8 at the $360 \mathrm{X}$ magnification, the microstructure of normalized and annealed samples comprises of both ferrite and pearlite in equal proportions. Their grain boundaries are not well defined but the grain boundaries of the annealed samples are coarser than that of the normalized one. The Figures reveals visible phases present in the steel and cracks are clearly visible along the grain boundaries of the sample. This is an indication that the heat treatment of the sample subjected it to some internal stresses which have caused cracks within the phases of the material. The quenching and tempering heat treatment process caused the formation of scattered grain particles which spread through in transgranular and intergranular spaces of the materials. Microstructural inspections indicated that the surfaces of tempered samples are martensitic.

Hardness Result. The results obtained from the hardness test are shown in Table 1. The table clearly show an improvement in hardness after hardening, and a decrease in hardness is observed with tempering. From the results the maximum hardness of $152 \mathrm{HBN}$ was obtained at $850^{\circ} \mathrm{C}$ hardening followed by the as receive carbon steel of about $131 \mathrm{HBN}$. The annealed carbon steel has the lowest hardness value of about $102 \mathrm{HBN}$.

Table 1. Hardness property of the as-received and the heat treated specimen

\begin{tabular}{|c|c|}
\hline Specimen Specification & Hardness \\
\hline As received & 131 \\
\hline Hardening & 152 \\
\hline Normalizing & 111 \\
\hline Tempering & 119 \\
\hline Annealing & 102 \\
\hline
\end{tabular}

Impact Test. Table 2 shows the energy recorded after an impact test for as-received specimen was $57.3 \mathrm{~J}$, while the heat treated specimen such as hardening, annealing, normalizing and tempering has the impact energy $64.1 \mathrm{~J}, 55.6 \mathrm{~J}, 65 \mathrm{~J}$, and $65.4 \mathrm{~J}$ respectively. From the impact test result, the tempered specimen has the highest impact energy just slightly greater than the normalized specimen which means that the tempered specimen can absorb more energy before failure more than the rest of the specimens and the annealed specimen has the lowest energy to absorb before failure.

Table 2. Impact test of the as-received and the heat treated specimen

\begin{tabular}{|l|l|}
\hline Specimen Specification & Toughness (J) \\
\hline As received & 57.3 \\
\hline Hardening & 64.1 \\
\hline Normalizing & 65 \\
\hline Tempering & 65.4 \\
\hline Annealing & 55.6 \\
\hline
\end{tabular}


Fatigue Result. Summary of fatigue test results used to compare between the heat treatment effects are presented in Table 3. The result shows that the endurance limit for normalized steel is higher than that of annealed one. As normalized materials have higher yield stress as compared to annealed one, the endurance limit is also higher than that of annealed one. Here for normalized treatment, fatigue limit comes around $604 \mathrm{MPa}$ for stress level at $1.1 \times 10^{3}$ cycles. But for annealed one, it came $604 \mathrm{MPa}$ at $1.4 \times 10^{3}$ no. of cycles. From the result at $604 \mathrm{MPa}$, the specimen with the highest numbers of cycle is the tempered at tempering temperature of $200^{0} \mathrm{C}$. When a load of about 1208 $\mathrm{MPa}$ was added there is a decrease in the number of cycle, while tempering exhibit the highest number of cycle but at the increment of load it decreases rapidly.

Table 3. Fatigue test results with different heat treatment conditions for SAE 1035 Steel specimens

\begin{tabular}{|l|l|l|}
\hline Specimen Specification & Stress (MPa) & No. of Cycles to Failure \\
\hline As received & 604 & $1.3 \times 10^{3}$ \\
\hline & 1208 & $0.7 \times 10^{3}$ \\
\hline & 1812.3 & $0.3 \times 10^{3}$ \\
\hline Normalizing & 604 & $1.1 \times 10^{3}$ \\
\hline & 1208 & $0.4 \times 10^{3}$ \\
\hline & 1812.3 & $0.2 \times 10^{3}$ \\
\hline Tempering & 604 & $2 \times 10^{3}$ \\
\hline & 1208 & $0.8 \times 10^{3}$ \\
\hline & 1812.3 & $0.1 \times 10^{3}$ \\
\hline Annealing & 604 & $1.4 \times 10^{3}$ \\
\hline & 1208 & $0.3 \times 10^{3}$ \\
\hline & 1812.3 & $0.1 \times 10^{3}$ \\
\hline
\end{tabular}

Tensile Result. The resulting engineering Load - Displacement curves obtained from the test are shown in Fig. 9 to Fig.13 for annealed, normalized, tempered and hardened and also the as receive specimens respectively. The test data are plotted against engineering stress (load) vs. engineering strain (elongation). Fig. 9 and Fig. 10 show that yield stress and ultimate tensile stress is more for normalized as compared to annealed treatment. This enhancement may be due to the fact that the cooling process is influenced by the cooling rate used for these treatments. Normalizing cooling rate compared to annealing is faster, because in normalizing cooling process is done by air cooling and in annealing this is done by furnace cooling. Due to this, more refine grains are obtained as compared to the annealed one which induces more strength and less ductility in the material [23].

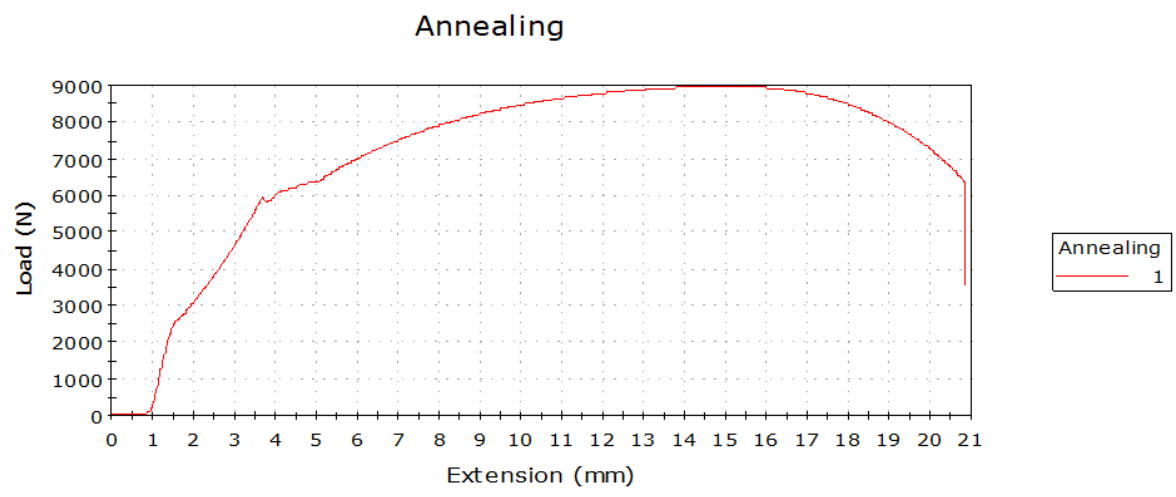

Fig. 9. Load - Displacement curve for the annealed specimen 


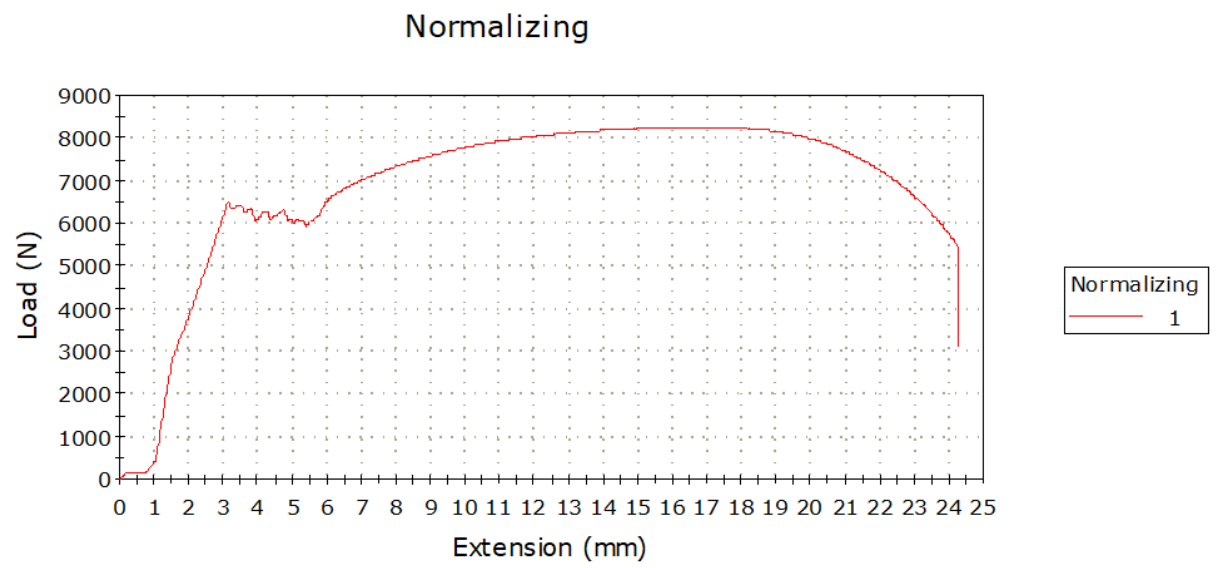

Fig. 10. Load - Displacement curve for the normalized specimen

Tempering

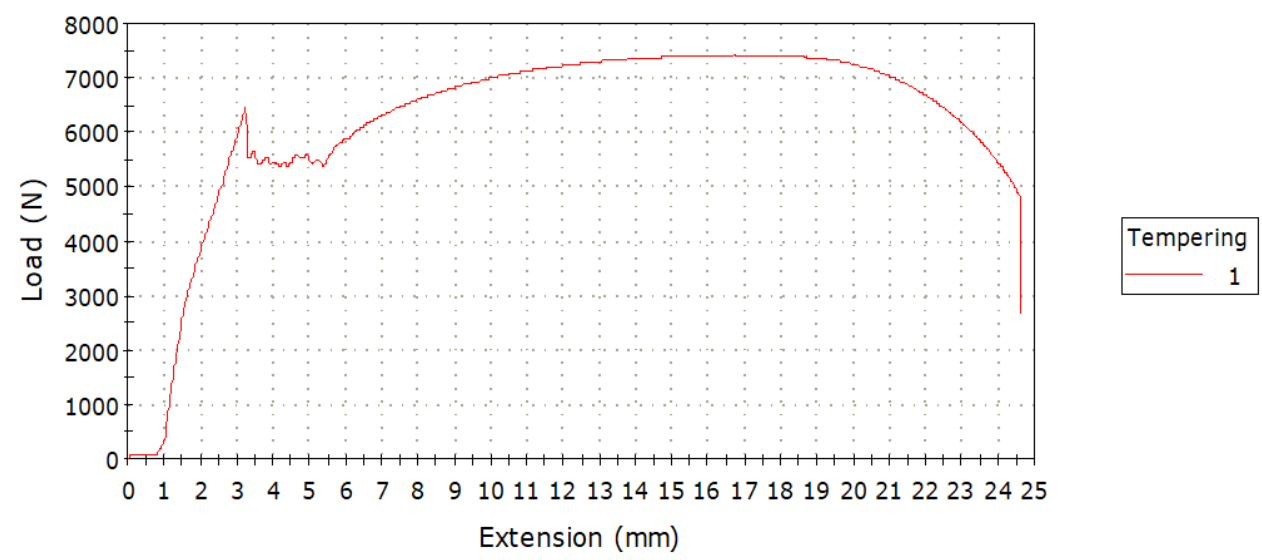

Fig. 11. Load - Displacement curve for the tempered specimen

From Fig. 11 compared to the normalizing and annealing treatments, tempering has higher value due to the more refined grains, as the specimens were subjected to austenizing, quenching and then tempering [14]. Another mechanical property variation which should be in consideration is ductility. During the tensile test, materials are elongated and then broken due to tensile stress. According to the tensile test of tempering $200{ }^{\circ} \mathrm{C}$ for higher time we get a result of $18 \mathrm{~mm} / \mathrm{mm} \%$ of elongation and for lower time $11 \mathrm{~mm} / \mathrm{mm}$, having less ductility as compared to the normalizing and annealing. From this we can conclude that when strength is induced in the materials, it makes the material more hard which gives the result of less ductility in the material.

\section{As Received}

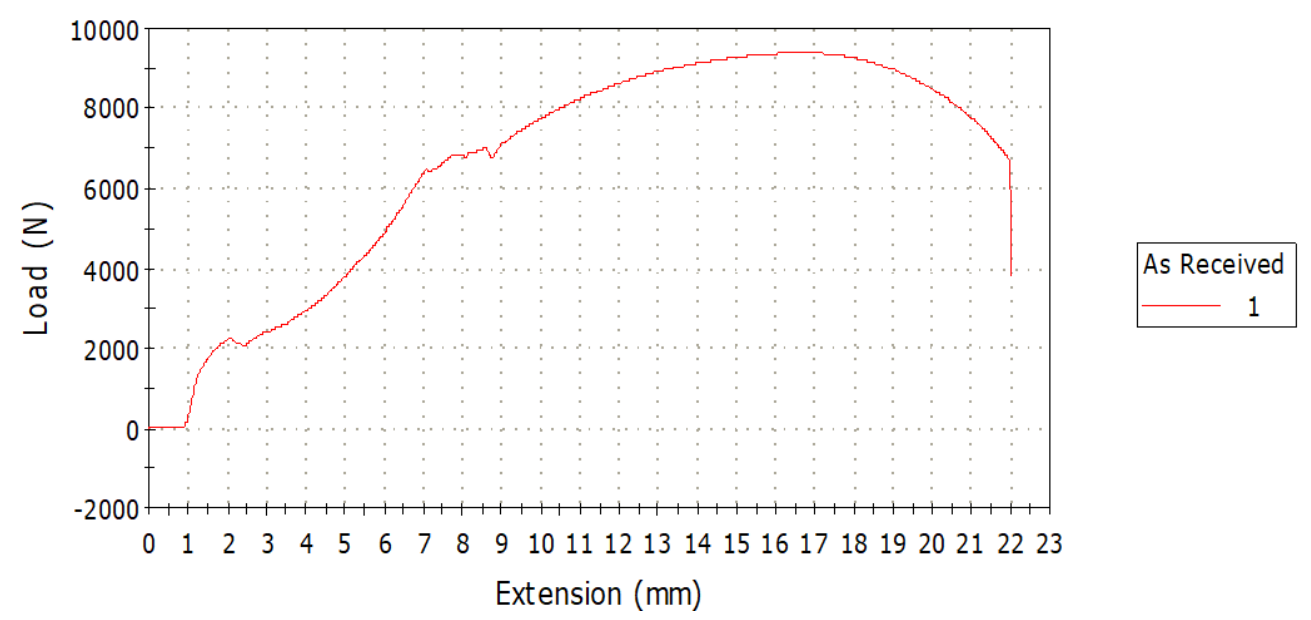

Fig. 12. Load - Displacement curve for the as receive specimen 


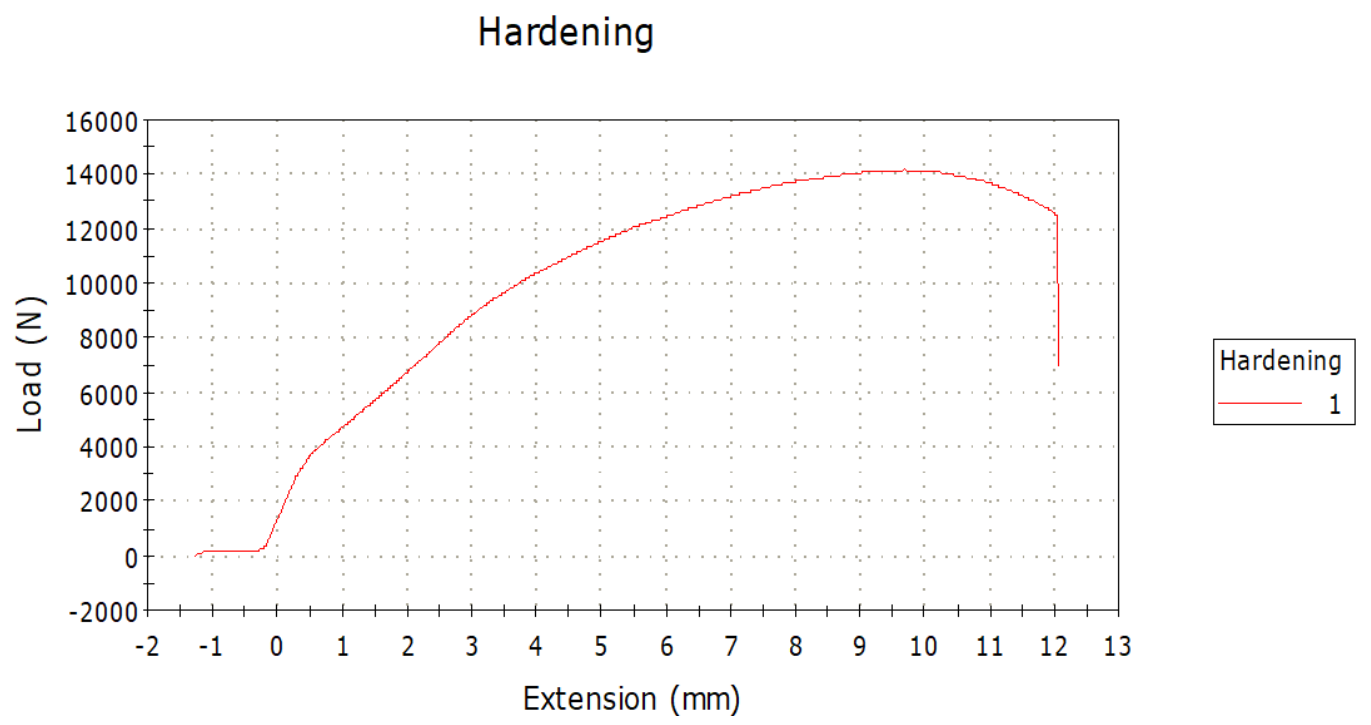

Fig. 13. Load - Displacement curve for the hardened specimen

In case of as-received specimen, the ultimate tensile strength, yield strength are $121 \mathrm{MPa}$ and $91.5 \mathrm{MPa}$ respectively, whereas the percentage elongation is $22 \mathrm{~mm} / \mathrm{mm}$. Comparing the as-receive with the hardened heat treated specimen, the hardened specimen has the highest ultimate tensile strength while the tempered specimen have the highest yield strength and the percentage elongation.

Modulus of Resilience. Table 4 shows the result of the modulus of resilience of the as received and the heat treated specimens. The Modulus of Resilience is the amount of energy stored in stressing the material to the elastic limit. Therefore the as receive specimen will have more stored energy when stressed to its elastic limit, followed by the hardened specimen which is slightly greater than the annealed specimen and the tempered specimen has the least stored energy when stress to its elastic limit more than the other specimen. The as received Specimen has a value of $2939 \mathrm{~N} / \mathrm{m}^{2}$, $1413 \mathrm{~N} / \mathrm{m}^{2}$ for the Annealed specimen and $1303 \mathrm{~N} / \mathrm{m}^{2}, 910 \mathrm{~N} / \mathrm{m} 21450 \mathrm{~N} / \mathrm{m}^{2}$ for the normalized, tempered and the hardened specimen respectively.

Table 4. The modulus of resilience of the as received and the heat treated specimen

\begin{tabular}{|l|l|}
\hline Specimen Specification & $\begin{array}{l}\text { Modulus of Resilience } \\
\text { (N/m) }\end{array}$ \\
\hline As received & 2939 \\
\hline Annealing & 1413 \\
\hline Normalizing & 1303 \\
\hline Tempering & 910 \\
\hline Hardening & 1450 \\
\hline
\end{tabular}

Young's Modulus. Table 5 Shows the young's modulus of the as-received and the heat treated specimen. From the figure, the annealed specimen has a young's modulus value of $399.5 \mathrm{MPa}$ followed by the hardened specimen with the value of $250.9 \mathrm{MPa}$ and that of the tempering, normalized and as-receive specimen are $267 \mathrm{MPa}, 203 \mathrm{MPa}$ and $116.3 \mathrm{MPa}$ respectively. The annealed specimen with the value of $399.5 \mathrm{MPa}$ can withstand more load than the other specimen in its elastic limit while the as receive specimen will withstand a lesser load to the other specimen.

Table 5. The Young's Modulus of the as received and the heat treated specimen

\begin{tabular}{|l|l|}
\hline Specimen Specification & Young's Modulus \\
\hline As received & 116.3 \\
\hline Annealing & 399.5 \\
\hline Normalizing & 203.8 \\
\hline Tempering & 267.1 \\
\hline Hardening & 250.9 \\
\hline
\end{tabular}


Ultimate Tensile Strength. Table 6 shows the ultimate tensile strength of the as-received and the heat treated specimen. Ultimate tensile strength (UTS), is the maximum stress that a material can withstand while being stretched or pulled before failing or breaking. From the figure, the annealed specimen having an ultimate strength of $118.6 \mathrm{MPa}$, and the as-receive specimen have an ultimate strength of $121 \mathrm{MPa}$ while the normalized, the tempered and the hardened specimen has the ultimate strength of 104.4 $\mathrm{MPa}, 95.5 \mathrm{M} \mathrm{Pa}$ and 179.6 MPa respectively. It can be seen from the figure that the hardened specimen has the highest value of ultimate strength of $179.6 \mathrm{MPa}$ and the tempered specimen has the lowest value with $95.5 \mathrm{MPa}$. Therefore, the hardened specimen will withstand more stress than the other specimen when stretched or pulled before failing or breaking, followed by the as-received, annealed and the normalized specimen and the tempered specimen will withstand the least stress than the other specimen when stretched or pulled.

Table 6. The Ultimate Tensile Strength of the as received and the heat treated specimen

\begin{tabular}{|l|l|}
\hline Specimen Specification & $\begin{array}{l}\text { Ultimate tensile strength } \\
\text { (MPa) }\end{array}$ \\
\hline As received & 121 \\
\hline Annealing & 118.6 \\
\hline Normalizing & 104.4 \\
\hline Tempering & 95.5 \\
\hline Hardening & 179.6 \\
\hline
\end{tabular}

Percentage Elongation. Table 7 shows the percentage elongation of the as-received and the heat treated specimen. The percentage elongation for the as receive and the heat treated specimen are as follows: as-received 22\%, Annealing 20.8\%, Normalizing 24.3\%, Tempering $24.6 \%$, Hardening $12 \%$. The tempered specimen having the highest elongation will be more ductile than the specimen slightly greater than the normalized specimen and the hardened specimen will be less ductile among the other specimen.

Table 7. The Percentage elongation of the as received and the heat treated specimen

\begin{tabular}{|l|l|}
\hline Specimen Specification & Percentage elongation (\%) \\
\hline As received & 22 \\
\hline Annealing & 20.9 \\
\hline Normalizing & 24.3 \\
\hline Tempering & 12 \\
\hline Hardening & 24.6 \\
\hline
\end{tabular}

Percentage Area Reduction. Table 8 shows the percentage area reduction of the as-received and the heat treated specimen. The as-received specimen have a percentage area reduction of $66 \%$, the Annealed specimen $81.5 \%$ while that of Normalized, Tempered and Hardened specimen are 59.1\%, $78.9 \%$ and $75 \%$ respectively. The Annealed specimen have the highest percentage area reduction followed by the Tempered specimen while the Normalized specimen have the least percentage area reduction. Therefore, the Annealed specimen will tend to have more increase in length during the necking period while the Normalized specimen with the least percentage area reduction will have lesser length increment during the necking period than the other specimen.

Table 8. The Percentage Area Reduction of the as received and the heat treated specimen

\begin{tabular}{|l|l|}
\hline Specimen Specification & Percentage Area Reduction (\%) \\
\hline As received & 66 \\
\hline Annealing & 81.5 \\
\hline Normalizing & 59.1 \\
\hline Tempering & 78.9 \\
\hline Hardening & 75 \\
\hline
\end{tabular}




\section{Conclusion}

In this study effect of different heat treatment process such as annealing, tempering, normalizing and hardening on mechanical properties such as impact, fatigue, hardness and tensile strength was investigated. From this study, it may be concluded that the increase of fatigue strength is directly proportional to increase in tensile strength. The best results are obtained for the specimen tempered at $200{ }^{0} \mathrm{C}$. These specimens have also shown the highest endurance limit. This is perhaps due to the fact that these specimen possess vary high strength with significant ductility. So, as far as fatigue strength is concerned, the tempering may be regarded as the best possible heat treatment operation. Among the heat treatment techniques, tempering has got the most significant effect on fatigue life. The changes caused by normalizing are mainly due to the increase in tensile strength in comparison with annealing. Tempering treatment improves fatigue behavior and also other mechanical properties. It can also be concluded that the material having higher UTS bear higher endurance limits as compared to materials with other treatments. Comparing the mechanical properties of tempering specimen with hardened sample, it was found that there was increase in young's modulus and hardness.

\section{Acknowledgement}

The authors wish to acknowledge the support given by Alo Francis of Materials and Metallurgical Engineering Department, Obafemi Awolowo University, Ife for providing facilities for this research. Also, the authors would like to thank the authors whose references are used for this research.

\section{References}

[1] M.A.S.Torres, H.J.C.Voorwald, An evaluation of shot peening, residual stress and stress relaxation on the fatigue life of AISI 4340 steel, Int. J. Fatigue. 24(8) (2002) 877-886. [doi:10.1016/S0142- 1123(01)00205-5]

[2] A. Fatemi, L .Yang, Cumulative Fatigue Damage, Life Prediction Theories: a Survey of the State of The Art for Homogeneous Material, International Journal of Fatigue. 20 (1998) 9-34.

[3] J.A. Bannantine, J.J. Comer, J.L. Handrock, Fundamentals of metal fatigue analysis, Prentice Hall, New York, 1990

[4] F. Gunnberg, M. Escursell, M. Jacobson, The influence of cutting parameters on residual stresses and surface topography during hard turning of $18 \mathrm{MnCr} 5$ case carburised steel, J Mater Proc Technol. 174 (2006) 82-90.

[5] D.Y. Wei, J.L. Gu, H.S. Fang, B.Z. Bai, Z.G. Yang, Fatigue Behavior of $1500 \mathrm{MPa}$ Bainite/Martensite Duplex-Phase High Strength Steel, International Journal of Fatigue. 26(4) (2004) 437-442.

[6] S. Zahid, P.P. Hector, A. Salam, J. Ahmad, Effect of different phase proportion of martensite on the mechanical properties of dual phase steel, Journal of Scientific Research. XXXIX (2) (2009).

[7] M.A. Maleque, Y.M. Poon H.H. Masjuki, The Effect of Intercritical Heat Treatment on the Mechanical Properties of AISI 3115 Steel, Journal of Materials Processing Technology. 153-154(13) (2004) 482-48.

[8] C. Adnan, Effect of Cooling rate on Hardness and Microstructure of AISI 1020, AISI 1040 and AISI 1060 Steels, Int. J. of Phys. Sci. 4(9) (2009) 514-518.

[9] G. Golañski, Effect of the heat treatment on the structure and properties of cast steel, Archives of material science and engineering. 46(2) (2010) 88-97. 
[10] K.K. Alaneme, I.M. Momoh, Mechanical Properties and Corrosion Sensitivity of Dual Phase Medium Carbon Low Alloy Steel, ANNALS of Faculty Engineering Hunedoara - International Journal of Engineering. 12 (2012).

[11] S.Z.Qamar, Effect of Heat Treatment on mechanical properties of H11 tool steel, Journal of Achievements in materials and manufacturing engineering, 35(2) (2009) 115-120.

[12] S.K. Saha, L. Prasad, V. Kumar, Experimental Investigations On Heat Treatment Of Cold Work Tool Steels: Part 1, International Journal of Engineering Research and Applications. 2(2) (2012) 510-519.

[13] S.Z. Qamar, Effect of Heat Treatment on Mechanical properties of H11 tool steel, Journal of Achievements in materials and manufacturing engineering. 35(2) (2009) 115-120.

[14] V.K.Murugan, P.Koshy Mathews, Effect of Tempering Behavior on Heat Treated Medium Carbon (C 35, Mn 75) Steel, International Journal of Innovative Research in Science, Engineering and Technology. 2(4) (2013). 